\title{
ANDES

www.scielo.cl

\section{Pancreatitis aguda asociada a infección por SARS-CoV-2 en un paciente pediátrico}

\author{
Acute pancreatitis associated with SARS-CoV-2 infection in a pediatric patient \\ De la Barra Sofía ${ }^{a}$, Izquierdo Giannina ${ }^{\mathrm{a}, \mathrm{b}}$, Rivacoba María Carolina ${ }^{\mathrm{b}}$, Román Gabriela ${ }^{\mathrm{c}}$, Piñera Cecilia $^{\mathrm{a}, \mathrm{b}}$
}

`Departamento de Pediatría y Cirugía Infantil, Campus Sur, Facultad de Medicina Universidad de Chile. Santiago, Chile
bUnidad de Infectología, Hospital Exequiel González Cortés. Santiago, Chile
'Unidad de Gastroenterología, Hospital Exequiel González Cortés. Santiago, Chile

Recibido: 9 de octubre de 2020; Aceptado: 4 de abril de 2021

\section{¿Qué se sabe del tema que trata este estudio?}

Si bien las manifestaciones posibles de la infección por SARS-CoV-2 son variadas, la descripción en la literatura de afectación pancreática asociada a esta infección es limitada.

\section{¿Qué aporta este estudio a lo ya conocido?}

Un nuevo caso de pancreatitis aguda con asociación temporal con la infección por SARS-CoV-2 en donde se excluyeron las etiologías más frecuentes y por lo tanto alerta de esta manifestación y posible relación causal.

\section{Resumen}

La infección por el nuevo coronavirus SARS-CoV-2 y su enfermedad COVID-19 ha representado un desafío para los clínicos, afectando distintos órganos y sistemas, principalmente el respiratorio. El compromiso pancreático está poco descrito, con escasos reportes en la literatura. Objetivo: comunicar un caso de pancreatitis aguda asociada a infección por SARS-CoV-2, con el propósito de contribuir al conocimiento de este nuevo virus y sus posibles formas de presentación. Caso Clínico: Adolescente masculino de 11 años, sin antecedente de contacto con personas confirmadas o sospechosas de COVID-19, que se hospitalizó por cuadro de dolor abdominal cólico, periumbilical y epigástrico, y vómitos frecuentes de 3 días de evolución, asociado a ausencia de deposiciones, sin otros síntomas asociados. En los exámenes de laboratorio destacó elevación de enzimas pancreáticas. La tomografía computada fue compatible con pancreatitis aguda edematosa intersticial sin signos de patología biliar. Se diagnosticó pancreatitis aguda al mismo tiempo que se aisló SARS-CoV-2 en el tracto respiratorio, sin identificarse otras posibles causas. El paciente de manejó en Unidad de Paciente Crítico con medidas de soporte y terapia, evolucionó favorablemente, sin síntomas respiratorios. Conclusión: El SARS-CoV-2 puede asociarse a presentaciones atípicas, entre ellas pancreatitis aguda. Aún no está claro cuál es el mecanismo fisiopatológico del daño pancreático. Los médicos deben estar atentos al compromiso por COVID-19 de otros sistemas, además del respiratorio.

Correspondencia:

Sofía De la Barra

sofia.delabarra.e@gmail.com 


\begin{abstract}
The recent discovery of SARS-CoV-2 and the disease COVID-19 which affects different organs and systems, mainly the respiratory one, representing a new challenge for physicians. Pancreatic affection is barely described, with only a few cases reported in the literature. Objective: to communicate a case of acute pancreatitis associated with SARS-CoV-2 infection, to contribute to the knowledge of this new virus and its possible forms of presentation. Clinical Case: An eleven-year-old male adolescent, with no history of contact with people confirmed or suspected of COVID-19, was admitted to the hospital with a 3-day history of periumbilical and epigastric abdominal pain, vomiting, and absence of stools, with no other symptoms. The laboratory tests showed increased pancreatic enzymes. Computed tomography was compatible with acute edematous pancreatitis, without signs of biliary pathology, diagnosing acute pancreatitis, at the same time that SARS-CoV-2 was isolated in the respiratory tract. Other possible differential diagnoses and history of epidemiological contact were ruled out. The patient was managed in the Critical Patient Unit with support measures. He evolved favorably, without respiratory symptoms. Conclusion: SARS-CoV-2 infection can be associated with atypical presentations, including acute pancreatitis. The physiopathological mechanism of pancreatic damage is not yet clear. Physicians should be aware of the COVID-19 involvement of other systems, beyond the respiratory one.
\end{abstract}

\section{Keywords:}

Pancreatitis;

Coronavirus;

SARS-CoV-2;

COVID-19;

Coronavirus Infections;

Pediatrics

\section{Introducción}

En diciembre de 2019 en Wuhan, China, fue detectado el primer caso de coronavirus SARS-CoV-2 y de acuerdo a los datos que entrega la Organización Mundial de la Salud (OMS), es responsable de una de las mayores pandemias del último siglo, con más de ciento veintidós millones de personas contagiadas y más de dos millones setecientas mil muertes hasta el 21 de marzo de 2021 $1^{1}$. En Chile hasta el 25 de marzo de 2021 han ocurrido 1.125.521 casos de COVID-19 (tasa aproximada de 5.622 casos por 100.000 habitantes), donde el 7,03\% correspondieron a menores de 15 años $^{2}$.

Investigadores alrededor de todo el mundo intentan revelar la mayor cantidad de información posible acerca de esta nueva enfermedad. Si bien en pacientes adultos su principal compromiso es del sistema respiratorio, en niños se ha descrito fiebre $(35-71 \%$ de los casos), tos (37-57\%), disnea (6,5-28,6\%), y las manifestaciones gastrointestinales en ellos alcanzan en promedio un $18 \%$ de frecuencia. Dentro de las manifestaciones gastrointestinales en la población pediátrica se ha descrito dolor abdominal $(6,7-7,7 \%)$, diarrea $(8,8-$ $21,4 \%)$, náuseas y/o vómitos $(6,4-10,4 \%)$, entre otras manifestaciones menos frecuentes como ictericia o elevación de transaminasas ${ }^{3-6}$. En Chile según el informe epidemiológico "Descripción epidemiológica de niños, niñas y adolescentes con COVID-19 Chile (semana epidemiológica 10 a 44, año 2020)", hasta el 01 de noviembre de 2020, de los 57.375 niños con COVID-19, el 9,5\% presentó diarrea y el 7,6\% dolor abdominal ${ }^{7}$.

Una revisión sistemática que incluyó 43 estudios con un total de 18.246 pacientes, describe la diarrea como el síntoma gastrointestinal más común, con un
$11,5 \%$, seguido por náuseas $(6,3 \%)$ y dolor abdominal $(2,3 \%)$. De aquellos con síntomas gastrointestinales que se les midieron transaminasas, se observó que un $31,4 \%$ presentó un leve ascenso de aspartato aminotransferasa y alanino aminotransferasa ${ }^{8}$.

Hasta el momento hay pocos casos reportados en la literatura sobre pancreatitis aguda asociada a infección por SARS-CoV-2 en adultos y escasos reportes en la población pediátrica ${ }^{9-14}$. Se ha descrito una gran similitud estructural entre SARS-CoV-2 con el SARS$\mathrm{CoV}$, lo que ha llevado a suponer que este nuevo virus infecta las células utilizando como receptor a la enzima convertidora de angiotensina $2(\text { ECA- } 2)^{15}$. Si bien es ampliamente conocida la localización pulmonar de este receptor, estudios clínicos han demostrado la expresión de ECA-2 en otros tejidos, dentro de ellos los islotes de Langerhans, convirtiendo al páncreas en un sitio hipotético de posible infección y daño ${ }^{16-18}$. En los últimos reportes se describe que SARS-CoV-2 utiliza el receptor ECA-2 para entrar a la célula en sinergia con la proteasa transmembrana de serina 2 (TMPRSS2) ubicadas en la superficie de las células del hospedero. Específicamente, la glicoproteína viral S es escindida por TMPRSS2, facilitando la activación viral, lo que representa uno de los factores claves del hospedero para la patogenicidad de SARS-CoV-2 ${ }^{19-20}$.

El objetivo de este artículo es comunicar un caso de pancreatitis aguda asociada a infección por SARSCoV-2 con el propósito de contribuir al conocimiento científico sobre este nuevo virus y sus posibles formas de presentación, buscando alertar a los médicos no especialistas, especialistas y subespecialistas de esta potencial presentación, para facilitar su detección y manejo precoz. 


\section{Caso Clínico}

Adolescente de 11 años, de sexo masculino, con antecedentes de constipación crónica, sobrepeso y vacunación completa correspondiente al programa nacional de inmunizaciones de Chile, sin antecedente de haber tenido contacto con personas confirmadas o sospechosas de COVID-19, ni viajes al extranjero. Consultó en mayo de 2020 en el servicio de urgencia del Hospital Doctor Exequiel González Cortés (HEGC) con un cuadro de 3 días de evolución caracterizado por dolor tipo cólico, periumbilical y epigástrico, asociado a rechazo alimentario y vómitos frecuentes (aproximadamente 10 episodios al día), además de ausencia de deposiciones por 2 días. No refirió fiebre en los días previos a su hospitalización, ni síntomas respiratorios.

$\mathrm{Al}$ ingreso al servicio de urgencia, el paciente se encontraba subfebril $\left(37,7^{\circ} \mathrm{C}\right.$ axilar), hipertenso (149/90 mmHg, > p99 para edad, estatura y sexo), sin apremio respiratorio, bien perfundido e hidratado, con un examen abdominal donde destacaba un abdomen distendido y sensible, sin signo de Blumberg. Se realizó una radiografía de abdomen (de pie postero anterior y decúbito lateral) y tacto rectal, siendo ambos normales, y fue evaluado por cirugía, a quienes no les impresionó como un abdomen agudo quirúrgico y se descartó una posible obstrucción intestinal. Sin embargo, por la intensidad y características del dolor abdominal se realizaron exámenes de laboratorio donde destacaba proteína C reactiva (PCR) elevada de $61 \mathrm{mg} / \mathrm{L}$ (valor normal $(\mathrm{VN})<5$ ), leucocitosis de $15.030 / \mathrm{uL}$ con predominio de polimorfonucleares $(84,6 \%)$ con linfopenia $(5,6 \%$, valor absoluto de $842 / \mathrm{uL})$, amilasa de 1.100 U/L (VN 28 - 100) y lipasa de 1.054 U/L (VN 13-60), con bilirrubina total de 1,3 gr/dL (34\% directa), hipoprotrombinemia (tiempo de protrombina $57 \%$ ), el resto de pruebas hepáticas dentro de rangos normales, perfil lipídico normal (triglicéridos $71 \mathrm{mg} / \mathrm{dL}$ ) y calcemia normal.

Se solicitó una tomografía computada (TC) de abdomen y pelvis con contraste (figura 1), donde se observó páncreas con leve aumento global en su volumen y pérdida del patrón acinar, con conducto pancreático principal prominente, con calibre de $3 \mathrm{~mm}$, compatible con pancreatitis aguda edematosa intersticial sin signos de patología biliar. De acuerdo a los criterios del Grupo Internacional de Estudio de la Pancreatitis Pediátrica (en inglés "International Study Group of Pediatric Pancreatitis" - INSPPIRE) ${ }^{21}$, se constituyó el diagnóstico de pancreatitis aguda cumpliendo con los 3 criterios (dolor abdominal, elevación del nivel sérico de lipasa y/o amilasa $\geq 3$ veces por sobre el límite normal y hallazgos imagenológicos compatibles).

Se hospitalizó en la Unidad de Paciente Crítico (UPC) para monitoreo, donde por protocolo de ingre- so hospitalario se tomó muestra de hisopado nasofaríngeo para detección de SARS-CoV-2 por técnica de reacción de la polimerasa en cadena con transcriptasa reversa (RPC-TR).

En UPC el paciente evolucionó subfebril, hipertenso, con dolor abdominal intenso que requirió bomba de infusión continua con fentanilo durante un día y ayuno prolongado $(>72 \mathrm{~h}$ ) por intolerancia oral. $\mathrm{Du}-$ rante el tercer día de hospitalización se conoció el resultado de RPC SARS-CoV-2 positiva, con exámenes de control que revelaron un descenso de enzimas pancreáticas y un ascenso de PCR progresivos (tabla 1).

Los exámenes realizados según el protocolo local para pacientes con COVID-19 resultaron alterados con un dímero D elevado (7.504 ng/mL; VN < 400), fibrinógeno y $\mathrm{LDH}$ normal. Se revisó TC inicial y ante sospecha de pancreatitis necrosante se tomaron hemocultivos y se inició terapia antibiótica endovenosa empírica con amikacina ( $15 \mathrm{mg} / \mathrm{kg} /$ día) y metronidazol (30 mg/kg/día) además de tromboprofilaxis con enoxaparina $(1 \mathrm{mg} / \mathrm{kg} /$ día por vía subcutánea) por elevación del dímero $\mathrm{D}$.

El sexto día de evolución del cuadro clínico presentó un peak febril $\left(38,2^{\circ} \mathrm{C}\right)$, con procalcitonina baja $(0,1 \mathrm{ng} / \mathrm{mL})$ sin recurrencia de la fiebre días posteriores. Ese mismo día se realizó una ecografía abdominal que no mostraba alteración de la vía biliar y una TC de tórax, abdomen y pelvis de control donde se observó que persistía el aumento de volumen del páncreas,

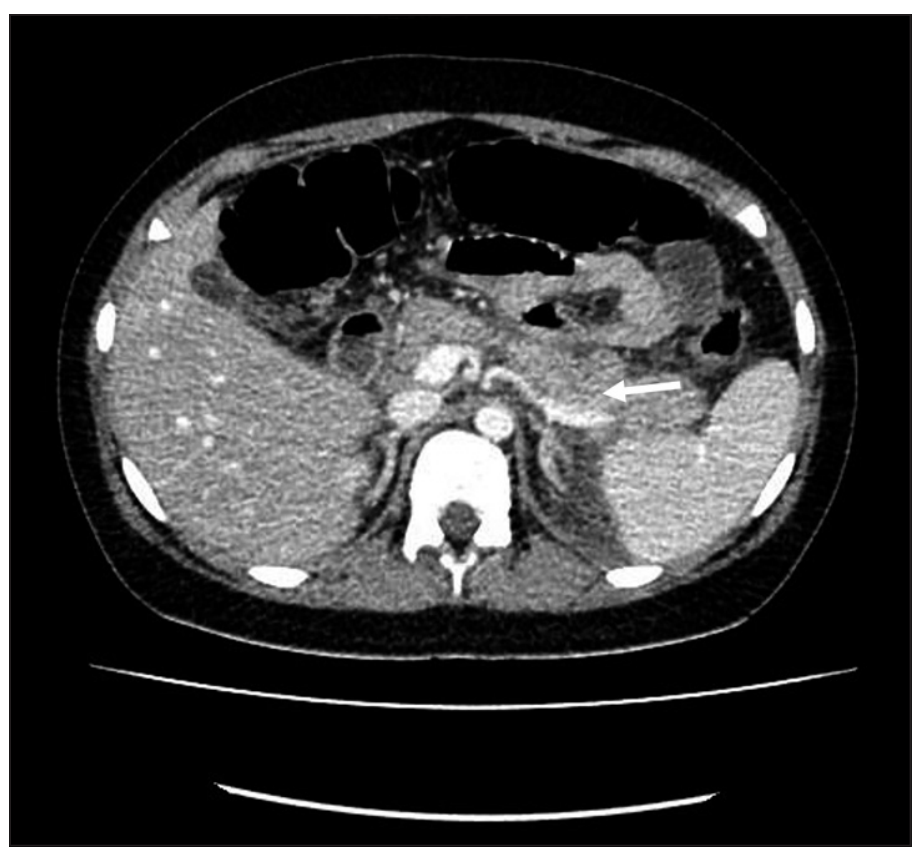

Figura 1. Tomografía axial computada de abdomen y pelvis con contraste correspondiente al primer día de hospitalización, compatible con pancreatitis aguda edematosa intersticial. Flecha blanca: parénquima pancreático aumentado de tamaño y con pérdida de patrón acinar. 
Tabla 1. Resumen de resultados de exámenes de laboratorio durante hospitalización

\begin{tabular}{lcccccc}
\hline & \multicolumn{5}{c}{ Días de hospitalización } \\
\hline Examen (Unidad, rango normal) & 1 & 2 & 3 & 4 & 5 & 6 \\
Proteína C reactiva ( $\leq 5 \mathrm{mg} / \mathrm{L})$ & 61,7 & 107,9 & 190,5 & 203,9 & 177,4 & 127 \\
Recuento glóbulos blancos (4.500-13.500/uL) & 15.030 & 10.630 & 11.820 & 12.320 & - & - \\
Amilasa (U/L, 28-100) & 1.100 & 467 & 230 & 147 & 120 & 115 \\
Lipasa (U/L, 13-60) & 1.054 & 444 & 203 & 226 & 88,6 & 124,8 \\
Dímero D (ng/mL, $\leq 500)$ & - & - & 7.504 & 5.658 & 2.829 & 3.928 \\
\hline
\end{tabular}

sin áreas de necrosis, sin colecciones, con mayor dilatación del conducto pancreático respecto a imagen previa ( 5 versus $3 \mathrm{~mm}$ ) y condensaciones atelectásicas en segmentos posteriores pulmonares, sin alteración del parénquima pulmonar compatible con COVID-19.

Para continuar con el diagnóstico diferencial de otras posibles causas de pancreatitis, durante el duodécimo día de evolución se realizó una colangioresonancia que mostró un conducto pancreático de diámetro normal, sin signos de colelitiasis ni coledocolitiasis, además de serología para citomegalovirus (CMV) con IgM negativa e IgG positiva que se interpretaron como una infección antigua; IgM anticápside de virus Epstein Barr (VEB) negativa y un panel molecular de virus respiratorios por hisopado nasofaríngeo negativo. Asimismo, no hubo desarrollo microbiológico en los hemocultivos tomados previo al inicio de antimicrobianos.

El paciente evolucionó afebril desde el quinto día de hospitalización, con mejoría de la tolerancia oral, sin dolor abdominal, sin síntomas respiratorios, sin requerir oxígeno en ningún momento, completando un total de siete días de antibioticoterapia empírica biasociada.

Luego del alta hospitalaria evolucionó en buenas condiciones generales, con episodios de dolor abdominal leve que cedían con paracetamol, se realizó seguimiento por vía telefónica en forma seriada y fue evaluado en policlínico de gastroenterología a los 14 días posterior al alta constatando un examen físico normal, descenso de las enzimas pancreáticas y un dímero D dentro de rangos normales.

\section{Discusión}

Presentamos un caso de pancreatitis aguda en un adolescente, asociada a SARS-CoV-2, sin otros agentes etiológicos identificados. Es discutible si el SARSCoV-2 es la causa de la pancreatitis o sólo una asociación temporal, en cuyo caso el diagnóstico sería pancreatitis idiopática y en forma concomitante una infección asintomática por SARS-CoV-2, ya que el paciente no tenía ninguna otra manifestación clínica. Sin embargo, se sabe que los virus pueden ser un agente causante de pancreatitis en aproximadamente $8-10 \%$ de los $\operatorname{casos}^{22}$. Respecto a la etiología de la pancreatitis, una variada lista de posibles causas fueron descartadas, tales como patología biliar, hipercalcemia, hipertrigliceridemia, hipotensión, alcohol, drogas, medicamentos, trauma, y otras infecciones virales. Teniendo esto en consideración se podría otorgar un rol a la infección por SARS-CoV-2 como posible agente causal de pancreatitis.

Chao y cols y Siegel y cols han reportado que el indicador más útil para el diagnóstico de pancreatitis aguda es la dilatación del conducto pancreático con una sensibilidad en pediatría por ecografía entre 78 a $92 \%$, con un alto valor predictivo positivo (86-91\%) y negativo (75-84\% $)^{23,24}$. Entonces, considerando que en nuestro caso clínico la colangioresonancia descartó la etiología obstructiva, la dilatación y posterior normalización del diámetro del conducto pancreático observados se explicarían por la evolución natural propia de la pancreatitis aguda.

Estudios previos han descrito la unión a ECA-2 asociado a TMPRSS2 como una de las principales vías de ingreso del SARS-CoV-2 a la célula ${ }^{15,18-20}$. Asimismo, hay autores que describen la presencia de ECA2 en diferentes tejidos, además del pulmonar ${ }^{16-18}$. En cortes histológicos pancreáticos, la proteína ECA-2 se ha visto expresada en la microvasculatura de tejido exocrino e islotes y en un subconjunto de conductos pancreáticos, mientras que la proteína TMPRSS2 se ha detectado restringida a las células ductales ${ }^{25}$. De esta forma SARS-CoV-2 podría afectar tejido pancreático.

Existe un reporte en la literatura que describe el aislamiento de SARS-CoV-2 por RPC en tejido pancreático, pero se desconoce si el mecanismo de daño del SARS-CoV-2 pudiera ser citopático directo en páncreas o mediado por procesos inflamatorios y/o inmunológicos ${ }^{26}$. Asimismo, no encontramos estudios que relacionaran carga viral de SARS-CoV-2 con la gravedad de la alteración pancreática. Faltan más estudios 
que aclaren la relación fisiopatológica entre SARSCoV-2 y la injuria pancreática.

En el contexto de que el SARS-CoV-2 pudiera asociarse a pancreatitis aguda, hasta el momento hay pocos estudios que registren esta relación. Destaca el escrito de Wang $\mathrm{F}$ y cols. realizado en Wuhan que incluyó 52 pacientes con COVID-19, de los cuales 17\% presentaron una alteración pancreática definida como elevación en los niveles de amilasa sérica ${ }^{12}$. En una cohorte retrospectiva de Mcnabb-Baltar J y cols, con 71 pacientes, $12,1 \%$ desarrollaron hiperamilasemia y solo 2 pacientes $(2,8 \%)$ superaron 3 veces el límite máximo normal, sin embargo, los autores concluyen que ninguno correspondió a una pancreatitis aguda propiamente $\mathrm{tal}^{27}$.

Los estudios mencionados han incluido exclusivamente pacientes adultos con COVID-19. Hasta el momento de esta comunicación, se han reportado escasos casos de pancreatitis asociado a COVID-19 en niños. El primer caso publicado corresponde a una niña de 7 años que luego de ser dada de alta con diagnóstico de pancreatitis, sin síntomas respiratorios, volvió a consultar dos semanas después por reaparición de clínica gastrointestinal con elevación de enzimas pancreáticas, que por contacto sospechoso se testeó y resultó positiva para SARS-CoV- $2^{13}$.

En Estados Unidos se reportó una serie de 3 casos; un niño de 11 años, otro de 15 años y una adolescente de 16 años. Al igual que nuestro caso, todos presentaron epigastralgia y rechazo alimentario, sin embargo, se reportó inicio de síntomas prodrómicos al menos 6 días antes del diagnóstico de pancreatitis. En esa serie destaca también la similitud del hemograma, con una linfopenia absoluta $(<1.500 / \mathrm{uL})$ y leucopenia $(<4.000 / \mathrm{uL})$. en solo uno de ellos. Los tres pacientes evolucionaron de manera favorable con una estadía hospitalaria de 3-4 días egresando con adecuada tolerancia oral y pruebas hepáticas y enzimas pancreáticas en vías de normalización ${ }^{14}$. Se debe agregar que en comparación, la cantidad de etiologías posibles descartadas en nuestro caso fue considerablemente mayor a las del reporte, destacando la dilatación del conducto pancreático como elemento de apoyo diagnóstico de pancreatitis aguda.

Inamdar Sumant y cols estudiaron las características epidemiológicas de pacientes hospitalizados con y sin COVID-19 con diagnóstico de pancreatitis en Northwell, Nueva York. Encontraron que la causa de la pancreatitis era idiopática en un mayor porcentaje de pacientes con COVID-19 comparado con quienes tenían PCR SARS-CoV-2 negativa (69\% vs $21 \%$, respectivamente); además observaron una mayor proporción de pacientes afrodescendientes e hispanos en aquellos con COVID-19 (OR 4,48 y 5,07, respectivamente). Los autores no observaron diferencias en mortalidad ni de- sarrollo de pancreatitis necrotizante entre aquellos con o $\sin$ SARS-CoV- $2^{28}$.

En cuanto a la temporalidad entre la infección por SARS-CoV-2 y la pancreatitis, en este caso se presume que correspondió a una infección aguda por coronavirus y que su única posible manifestación fue el compromiso gastrointestinal y pancreático, ya que no presentó sintomatología respiratoria. Si bien es sabido que el síndrome inflamatorio multisistémico pediátrico asociado a COVID-19 (SIM-C) puede comprometer múltiples sistemas, entre ellos el hematológico, renal, hemodinámico, cardiovascular, y el gastrointestinal (incluido páncreas) hasta en un $92 \%$ de los pacientes ${ }^{29}$, en este paciente no se consideró como diagnóstico el SIM-C, principalmente por la ausencia de fiebre persistente (de acuerdo a los criterios diagnósticos de la OMS) entre otros elementos, y, porque además este caso ocurrió en forma precoz en la pandemia, pocos días después del primer reporte de alerta de SIM-C del Royal College of London en Mayo de 2020, y no se disponía aún de serología para SARS-CoV2 en nuestro establecimiento para tener más elementos que apoyaran el diagnóstico diferencial ${ }^{30,31}$.

Se ha descrito que la temporalidad de la infección por SARS-CoV-2 y el desarrollo de pancreatitis aguda es heterogénea; algunos pacientes desarrollan síntomas de COVID-19 y dolor abdominal al inicio de la infección, sin embargo, se ha descrito otros casos en que la pancreatitis se desarrolla varios días después del diagnóstico de COVID-1932.

Aquí se reporta un caso de un adolescente de sexo masculino con cuadro clínico, exámenes de laboratorio e imágenes compatibles con pancreatitis aguda, descartando diversas etiologías, restando solamente infección viral por SARS-CoV-2 en forma contemporánea al diagnóstico. Esta posibilidad hace necesario que los médicos presten atención a la pancreatitis aguda y la consideren dentro de las posibles manifestaciones atípicas de COVID-19, tanto para aumentar la sospecha clínica, manejo oportuno, como para aumentar los reportes y mejorar así la casuística necesaria para develar esta posible asociación.

\section{Conclusión}

Hasta el momento existe poca información disponible respecto a la afectación pancreática asociada a infección por SARS-CoV-2 en adultos y escasa en población pediátrica. Futuros estudios podrían ayudar a precisar la relación entre estos fenómenos, por lo que se hace relevante advertir a los profesionales de la salud sobre la posible relación y la necesidad de considerar la pancreatitis aguda como presentación atípica de COVID-19. 


\section{Responsabilidades Éticas}

Protección de personas y animales: Los autores declaran que los procedimientos seguidos se conformaron a las normas éticas del comité de experimentación humana responsable y de acuerdo con la Asociación Médica Mundial y la Declaración de Helsinki.

Confidencialidad de los datos: Los autores declaran que han seguido los protocolos de su centro de trabajo sobre la publicación de datos de pacientes.
Derecho a la privacidad y consentimiento informado: Los autores han obtenido el consentimiento informado de los pacientes y/o sujetos referidos en el artículo. Este documento obra en poder del autor de correspondencia.

\section{Conflicto de intereses}

Las autoras declaran no tener conflicto de intereses.

\section{Referencias}

1. Organización Mundial de la Salud. Coronavirus disease (COVID-19), weekly epidemiological update. 23 Marzo 2021. Disponible en: https://www. who.int/publications/m/item/weeklyepidemiological-update-on-covid-19--23-march-2021, último acceso 28 de marzo 2021.

2. Departamento de Epidemiología, Ministerio de Salud de Chile. Informe epidemiológico $\mathrm{N}^{\circ} 106$. Enfermedad por SARS-CoV-2 (COVID-19). 28 Marzo 2021. Disponible en: https://www.minsal.cl/ wp-content/uploads/2021/03/Informeepidemiolo\%CC\%81gico-106-.pdf, último acceso 28 de marzo 2021.

3. Stokes EK, Zambrano LD, Anderson KN, et al. Coronavirus Disease 2019 Case Surveillance - United States, January 22-May 30, 2020. MMWR Morb Mortal Wkly Rep. 2020;69:759.

4. Cheung KS, Hung I, Chan P, et al. Gastrointestinal Manifestations of SARSCoV-2 Infection and Virus Load in Fecal Samples From a Hong Kong Cohort: Systematic Review and Meta-analysis. Gastroenterology 2020;159(1):81-95.

5. Liguoro I, Pilotto C, Bonanni M, et al. SARS-COV-2 infection in children and newborns: a systematic review. Eur J Pediatr. 2020;179:1029.

6. Hoang A, Chorath K, Moreira A, et al. COVID-19 in 7780 pediatric patients: A systematic review. EClinicalMedicine 2020;24:100433.

7. Departamento de Epidemiología, Ministerio de Salud de Chile. Descripción epidemiológica de niños, niñas y adolescentes con COVID-19 (semana epidemiológica 10 a 44). Chile 01 noviembre 2020. Disponible en: https://www.minsal.cl/wp-content/ uploads/2020/11/NI\%C3\%91OS_ COVID_01112020-AP_JA.pdf, último acceso 07 de enero 2021.

8. Silva F, Bittencourt de Brito B, Cordeiro ML, et al. COVID-19 gastrointestinal manifestations: a systematic review. Rev. Soc. Bras. Med. Trop. 2020;53:e20200714.

9. Hadi A, Werge M, Kristiansen KT, et al. Coronavirus Disease-19 (COVID-19) associated with severe acute pancreatitis: Case report on three family members. Pancreatology. 2020;20(4):665-7.

10. Meireles PA, Bessa F, Gaspar P, et al. Acalculous Acute Pancreatitis in a COVID-19 Patient. Eur J Case Rep Intern Med. 2020;7(6):001710.

11. Aloysius MM, Thatti A, Gupta A, et al. COVID-19 presenting as acute pancreatitis. Pancreatology. 2020;S14243903(20)30154-X.

12. Wang F, Wang H, Fan J, et al. Pancreatic Injury Patterns in Patients With Coronavirus Disease 19 Pneumonia. Gastroenterology. 2020;S00165085(20)30409-1.

13. Alloway BC, Yaeger SK, Mazzaccaro RJ, et al. Suspected case of COVID-19associated pancreatitis in a child. Radiol Case Rep. 2020;15(8):1309-12.

14. Samies NL, Yarbrough A, Boppana S. Pancreatitis in Pediatric Patients with COVID-19. Pediatr Infect Dis J, 2020;10(1):57-9.

15. Zhang $\mathrm{H}$, Penninger JM, Li Y, et al. Angiotensin-converting enzyme 2 (ACE2) as a SARS-CoV-2 receptor: molecular mechanisms and potential therapeutic target. Intensive Care Med. 2020;46(4):586-90.

16. Tahmasebi M, Puddefoot JR, et al. The tissue renin-angiotensin system in human pancreas. Int J Endocrinol. 1999;161(2):317-22.

17. Lam KY, Leung PS. Regulation and expression of a renin-angiotensin system in human pancreas and pancreatic endocrine tumours. Eur J Endocrinol. 2002;146(4):567-72.

18. Liu F, Long X, Zou W, et al. ACE2 Expression in Pancreas May Cause Pancreas Damage After SARS-CoV-2 Infection. Clin Gastroenterol Hepatol. 2020;18(9):2128-30.e2.

19. Cano F, Gajardo M, Freundlich M.
Eje Renina Angiotensina, Enzima Convertidora de Angiotensina 2 y Coronavirus. Rev Chil Pediatr. 2020;91(3):330-8.

20. Mollica V, Rizzo A, Massari F. The pivotal role of TMPRSS2 in coronavirus disease 2019 and prostate cancer. Future Oncol. 2020;16(27):2029-33.

21. Abu-El-Haija M, Kumar S, Quiros JA, et al. Management of Acute Pancreatitis in the Pediatric Population: A Clinical Report From the North American Society for Pediatric Gastroenterology, Hepatology and Nutrition Pancreas Committee. J Pediatr Gastroenterol Nutr. 2018;66(1):159-76.

22. Pohl JF, Uc A. Paediatric pancreatitis. Curr Opin Gastroenterol. 2015;31(5):3806.

23. Chao HC, Lin SJ, Kong MS, et al. Sonographic evaluation of the pancreatic duct in normal children and children with pancreatitis. J Ultrasound Med. 2000;19:757-63.

24. Siegel MJ, Martin KW, Worthington JL. Normal and abnormal pancreas in children: US studies. Radiology. 1987;165:15-8.

25. Coate KC, Cha J, Shrestha S, et al. SARS-CoV-2 Cell Entry Factors ACE2 and TMPRSS2 Are Expressed in the Microvasculature and Ducts of Human Pancreas but Are Not Enriched in Cells. Cell Metab. 2020;32(6):1028-40.e4.

26. Schepis T, Larghi A, Papa A, et al. SARSCoV2 RNA detection in a pancreatic pseudocyst sample. Pancreatology. 2020;20(5):1011-2.

27. McNabb-Baltar J, Jin DX, Grover AS, et al. Lipase Elevation in Patients With COVID-19. Am J Gastroenterol. 2020;115(8):1286-8.

28. Inamdar S, Benias PC, Liu Y, et al. Prevalence, Risk Factors, and Outcomes of Hospitalized Patients With Coronavirus Disease 2019 Presenting as Acute Pancreatitis. Gastroenterology. 2020;159(6):2226-8.e2.

29. Ahmed M, Advani S, Moreira A, et al. Multisystem inflammatory syndrome 
in children: A systematic review. EClinicalMedicine. 2020;26:100527.

30. World Health Organization. Multisystem inflammatory syndrome in children and adolescents with COVID-19- Scientific brief. 2020. Disponible en: https://www. who.int/publications/i/item/multisysteminflammatory-syndrome-in-childrenand-adolescents-with-covid-19. Último acceso 14 enero 2021.

31. Riphagen S, Gomez X, Gonzalez-Martinez $\mathrm{C}$, et al. Hyperinflammatory shock in children during COVID-19 pandemic Lancet. 2020;395(10237):1607-8.

32. de-Madaria E, Capurso G. COVID-19 and acute pancreatitis: examining the causality. Nat Rev Gastroenterol Hepatol. 2021;18(1):3-4 